\title{
Universal Finite-Size Scaling Function of the Ferromagnetic Heisenberg Chain in a Magnetic Field. II 8
}

\section{— Nonlinear Susceptibility —}

\author{
Hiroaki NAKAmURA, Naomichi Hatano ${ }^{1}$ and Minoru TAKAHASHI \\ Institute for Solid State Physics, University of Tokyo, \\ Roppongi, Minato-ku, Tokyo 106 \\ ${ }^{1}$ Department of Physics, University of Tokyo, \\ Hongo, Bunkyo-ku, Tokyo 113, \\ and Department of Physics, Harvard University, \\ Cambridge, MA 02138, USA
}

(Received

The finite-size scaling function of the nonlinear susceptibility of the ferromagnetic Heisenberg chain is given explicitly. It is conjectured that the scaling function is universal for any values of $S$. The conjecture is based on the exact solution of the nonlinear susceptibility for $S=\infty$, and numerical calculations for $S=1 / 2$ and $S=1$

KEYWORDS: Heisenberg chain, Heisenberg ferromagnet, numerical calculation, finite-size scaling function, universality, nonlinear susceptibility

* Submitted to J.Phys.Soc.Jpn 


\section{INTRODUCTION}

Thanks to recent development of technology, it has become possible to synthesize many quasi-low-dimensional systems experimentally. This has led to growing theoretical interest in low-temperature properties of lowdimensional systems. A remarkable example is the Haldane conjecture [1,2] for the spin- $S$ antiferromagnetic Heisenberg chain.

Recently, two of the present authors considered [3] low-temperature low-magnetic-field properties of the spin- $S$ ferromagnetic Heisenberg chain. The Hamiltonian of the model is defined by

$$
\mathcal{H}=-\frac{J}{S^{2}} \sum_{i=1}^{L} \boldsymbol{S}_{i} \cdot \boldsymbol{S}_{i+1}-\frac{h}{S} \sum_{i=1}^{L} S_{i}^{z},
$$

where the coupling constant $J$ is positive and $h$ denotes a magnetic field. They calculated [3] the free energy and the magnetization of the $S=1 / 2$ system numerically, employing the thermal Bethe-ansatz method [4 for the thermodynamic limit $L \rightarrow \infty$. They found [3] that the magnetization for the $S=1 / 2$ system has the same scaling function as that of the classical $(S=\infty)$ ferromagnetic Heisenberg chain:

$$
\begin{aligned}
m(T, h) & \simeq \tilde{m}_{0}(x), \\
& =\frac{2}{3} x-\frac{44}{135} x^{3}+\mathrm{O}\left(x^{5}\right) .
\end{aligned}
$$

Here $x$ is the scaling variable:

$$
x \equiv \frac{J h}{T^{2}}
$$

They thereby conjectured that this scaling function should be universal, or common to all values of $S$. This conjecture implies that the spinwave excitation from the ground state of the ferromagnet show universal behavior.

In order to confirm this conjecture for $1 \leq S<\infty$, we have to obtain information from numerical calculations of finite systems, because the Bethe ansatz method is not applicable to higher-spin Heisenberg chains. In the previous paper, [5] we hence generalized the above scaling form 
(1.2) to finite-size scaling and conjectured that the scaling function of the magnetization,

$$
m(T, h, L) \simeq \tilde{m}(x, y)
$$

is universal for the arbitrary- $S$ ferromagnetic Heisenberg chain. Here the scaling parameters $x$ and $y$ are defined by (1.4) and

$$
y \equiv \frac{J}{T L}
$$

It is difficult to obtain the magnetization $m(T, h, L)$ for the classical system. Hence, as the first step, we treated [5] the finite-size scaling form of the linear susceptibility. The scaling form (1.5) is followed by the finite-size scaling function of the linear susceptibility $\chi_{1}$ in the form

$$
\left.\chi_{1}(T, L) \equiv \frac{\partial m}{\partial h}\right|_{h=0} \simeq \frac{J}{T^{2}} \tilde{\chi}_{1}\left(\frac{J}{T L}\right),
$$

where

$$
\left.\tilde{\chi}_{1}(y) \equiv \frac{\partial \tilde{m}(x, y)}{\partial x}\right|_{x=0} .
$$

In the previous paper, [5] we analytically obtained the function $\tilde{\chi}_{1}$ for the classical, or the $S=\infty$ Heisenberg chain with both periodic and open boundary conditions. Moreover, we showed that the function $\tilde{\chi}_{1}$ for $S=\infty$ fits numerical data for $S=1 / 2$ and $S=1$ quite well. Thus we partly confirmed the conjecture on the universality of the scaling function (1.5).

In the present paper, we further treat the scaling function of the nonlinear susceptibility, and show its universality. Equation (1.5) ensures the universality not only of the linear susceptibility $\chi_{1}$ but also of the third-order nonlinear susceptibility $\chi_{3}$ :

$$
\left.\chi_{3}(T, L) \equiv \frac{1}{3 !} \frac{\partial^{3} m}{\partial h^{3}}\right|_{h=0} \simeq \frac{J^{3}}{T^{6}} \tilde{\chi}_{3}\left(\frac{J}{T L}\right),
$$

where 


$$
\left.\tilde{\chi}_{3}(y) \equiv \frac{1}{3 !} \frac{\partial^{3} \tilde{m}(x, y)}{\partial x^{3}}\right|_{x=0} .
$$

The result (1.3) in the thermodynamic limit requires the relation

$$
\tilde{\chi}_{3}(y=0)=-\frac{44}{135} \text {. }
$$

In the present paper we obtain an analytical expression of $\tilde{\chi}_{3}$ for the classical chain. (This was partially reported in the previous paper. [5]) We then show that the function $\tilde{\chi}_{3}$ obtained for $S=\infty$ is consistent with numerical data for quantum systems, namely, $S=1 / 2$ and $S=1$. Thus we confirm the universality of the scaling function (1.5) up to the third order of $x$.

In the course of the study, we also obtain the exact expressions of the four-point correlations and the nonlinear susceptibility of the classical system. To our knowledge, these expressions for the periodic classical system have not appeared in the literature.

Before going into details of calculations, we comment here on the definition of the scaling limit. We see in the following that there are corrections of the form $y \sqrt{T}, y T$, etc. to the finite-size scaling functions (1.7) and (1.9). These are less singular than the leading term of $y$ for large systems at low temperatures. We have to exclude these correction terms in order to obtain the scaling function of $y$. We hence extrapolate the limit $T \rightarrow 0$ while fixing the scaling variable $y=J /(L T)$. We refer to this limit as the scaling limit. It should not be confused with the thermodynamic limit $y \rightarrow 0$, or the limit $L \rightarrow \infty$ with $T$ fixed. Even after taking the scaling limit, we still consider a finite value of $y=J /(T L)$.

We organize the paper as follows. In $\S 2$ we analytically calculate the finite-size scaling function of $\chi_{3}$ of the classical Heisenberg model for the periodic and open boundary conditions. We show numerical confirmation of the universality of the scaling function for $S=1 / 2$ and $S=1 \mathrm{in} \S 3$. In $\S 4$ we discuss origins of the universality of the third-order nonlinear susceptibility. In Appendix A we present in detail the analytic calculation of the four-point correlation function of the classical Heisenberg chain with periodic boundary condition. In Appendix B we show the derivation of the third-order nonlinear susceptibility of the classical Heisenberg chain. 


\section{FINITE-SIZE SCALING FUNCTIONS OF THE THIRD-ORDER NONLINEAR SUSCEPTIBILITY}

In the previous paper [5] we presented the finite-size scaling function of the linear susceptibility, $\tilde{\chi}_{1}$. We used the classical Heisenberg chain to obtain the analytic form of $\tilde{\chi}_{1}$. In this section we derive the finite-size scaling function of the third-order nonlinear susceptibility, $\tilde{\chi}_{3}$, analytically for the classical Heisenberg chain with the periodic and the open boundary conditions. We insist that the ferromagnetic Heisenberg chain has this finite-size scaling function $\tilde{\chi}_{3}$ not only in the classical case $(S=\infty)$ but also in the quantum case.

The classical Heisenberg chain is given by

$$
\begin{aligned}
\mathcal{H} & =-\frac{J}{S^{2}} \sum_{i=1}^{L} \boldsymbol{S}_{i} \cdot \boldsymbol{S}_{i+1}-\frac{h}{S} \sum_{i=1}^{L} S_{i}^{z} \\
& \rightarrow-J \sum_{i=1}^{L} \boldsymbol{n}_{i} \cdot \boldsymbol{n}_{i+1}-h \sum_{i=1}^{L} n_{i}^{z} \quad \text { as } \quad S \rightarrow \infty,
\end{aligned}
$$

where $\left\{\boldsymbol{n}_{i}\right\}$ are vectors of length unity. The nonlinear susceptibility is given by summation of four-point correlations as follows:

$$
\begin{aligned}
\chi_{3}(T, L)= & \frac{1}{3 ! L T^{3} S^{4}} \sum_{i_{1}, i_{2}, i_{3}, i_{4}}\left[\left\langle S_{i_{1}}^{z} S_{i_{2}}^{z} S_{i_{3}}^{z} S_{i_{4}}^{z}\right\rangle\right. \\
& -\left\langle S_{i_{1}}^{z} S_{i_{2}}^{z}\right\rangle\left\langle S_{i_{3}}^{z} S_{i_{4}}^{z}\right\rangle \\
& -\left\langle S_{i_{1}}^{z} S_{i_{3}}^{z}\right\rangle\left\langle S_{i_{2}}^{z} S_{i_{4}}^{z}\right\rangle \\
& \left.-\left\langle S_{i_{1}}^{z} S_{i_{4}}^{z}\right\rangle\left\langle S_{i_{3}}^{z} S_{i_{2}}^{z}\right\rangle\right]
\end{aligned}
$$

where $\langle\cdots\rangle$ denotes the thermal average.

\section{A. Periodic boundary condition}

First, we consider the periodic system. To obtain the nonlinear susceptibility, we have to calculate the partition function, the two-point correlation function and the four-point correlation function. 
Joyce [6] obtained the partition function of the periodic chain, $Z_{L}$, in the form

$$
Z_{L}(K)=\sum_{l=0}^{\infty}(2 l+1) \lambda_{l}(K)^{L}
$$

where $K \equiv J / T$ and

$$
\lambda_{l}(K) \equiv \sqrt{\frac{\pi}{2 K}} I_{l+\frac{1}{2}}(K)
$$

with $I_{l+\frac{1}{2}}(K)$ being modified Bessel functions of the first kind. The twopoint correlation function schematically shown in Fig. 11 is given by [6,5]

$$
\left\langle n_{1}^{z} n_{1+M}^{z}\right\rangle_{L}^{\text {peri }}=\frac{1}{3 Z_{L}} \sum_{l=0}^{\infty}(l+1) \lambda_{l}^{L}\left[u_{l}^{M}+u_{l}^{L-M}\right]
$$

where the bracket $\langle\cdots\rangle_{L}^{\text {peri }}$ denotes the thermal average for the periodic system of length $L$, and the function $u_{l}$ is defined by

$$
u_{l}(K) \equiv \frac{\lambda_{l+1}(K)}{\lambda_{l}(K)}
$$

From the calculations given in Appendix A, we also obtain the four-point correlation function shown in Fig. 2 as

$$
\begin{aligned}
& \left\langle n_{1}^{z} n_{1+A}^{z} n_{1+A+B}^{z} n_{1+A+B+C}^{z}\right\rangle_{L}^{\text {peri }} \\
& =\frac{1}{Z_{L}} \sum_{l=0}^{\infty} \lambda_{l}^{L}\left\{f_{l}\left[u_{l}^{A} v_{l}^{B} u_{l}^{C}+u_{l}^{B} v_{l}^{C} u_{l}^{D}+u_{l}^{C} v_{l}^{D} u_{l}^{A}+u_{l}^{D} v_{l}^{A} u_{l}^{B}\right]\right. \\
& \left.\quad+g_{l}\left[u_{l}^{A} u_{l}^{C}+u_{l}^{B} u_{l}^{D}\right]\right\}
\end{aligned}
$$

where $D=L-A-B-C$,

$$
\begin{aligned}
f_{l} & \equiv \frac{2}{15} \frac{(l+1)(l+2)}{2 l+3} \\
g_{l} & \equiv \frac{1}{15} \frac{(l+1)\left(4 l^{2}+8 l+5\right)}{(2 l+1)(2 l+3)}
\end{aligned}
$$

and 


$$
v_{l}(K) \equiv \frac{\lambda_{l+2}(K)}{\lambda_{l}(K)}
$$

These expressions (2.3)-(2.10) give the exact expression of the nonlinear susceptibility (2.2). See Appendix B for details. Now we calculate its scaling form.

In the scaling limit, that is, in the limit $T \rightarrow 0$ with $y=J /(T L)$ fixed, the partition function is expressed in terms of $y$ as follows: [5]

$$
\begin{aligned}
& \frac{Z_{L}(K)}{\lambda_{0}(K)^{L}}=\sum_{l=0}^{\infty}(2 l+1)\left(\frac{\lambda_{l}}{\lambda_{0}}\right)^{L} \\
& \rightarrow \tilde{\mathcal{W}}(y) \equiv \sum_{l=0}^{\infty}(2 l+1) \exp \left\{-\frac{l(l+1)}{2 y}\right\} .
\end{aligned}
$$

Next, we can obtain the finite-size scaling function (1.9) of the third-order susceptibility with the periodic boundary condition, $\tilde{\chi}_{3}^{\text {peri }}$, as follows:

$$
\begin{aligned}
\tilde{\chi}_{3}^{\text {peri }}(y)= & \lim _{\substack{T \rightarrow 0 \\
y \text { fixed }}}\left[\frac{\chi_{3}^{\text {peri }}(T, L) T^{6}}{J^{3}}\right] \\
= & \frac{1}{\tilde{\mathcal{W}}(y)} \sum_{l=0}^{\infty} \exp \left[-\frac{l(l+1)}{2 y}\right]\left\{\frac{8}{15} \frac{l+2}{2 l+3}\right. \\
& \times\left[-\frac{1}{(l+2) y} \exp \left(-\frac{l+1}{y}\right)\right. \\
& +\frac{1}{(2 l+3)(l+1)}\left(1-\exp \left(-\frac{l+1}{y}\right)\right) \\
& \left.-\frac{l+1}{(2 l+3)(l+2)^{2}}\left(\exp \left(-\frac{2 l+3}{y}\right)-\exp \left(-\frac{l+1}{y}\right)\right)\right] \\
& +\frac{2}{15} \frac{4 l^{2}+8 l+5}{(2 l+1)(2 l+3)} \\
& \times\left[\frac{1}{(l+1) y}\left(1+\exp \left(-\frac{l+1}{y}\right)\right)\right. \\
& \left.\left.-\frac{2}{(l+1)^{2}}\left(1-\exp \left(-\frac{l+1}{y}\right)\right)\right]\right\} \\
& -\frac{1}{\tilde{\mathcal{W}}(y)^{2}} \frac{2}{9 y} .
\end{aligned}
$$


The key in the calculations of the above scaling forms is to note the behavior of the functions $u_{l}$ and $v_{l}$. These functions behave at low temperatures as follows: [0]

$$
\begin{aligned}
& u_{l}(K) \rightarrow 1-\frac{l+1}{K}+O\left(\frac{1}{K^{2}}\right), \\
& v_{l}(K) \rightarrow 1-\frac{2 l+3}{K}+O\left(\frac{1}{K^{2}}\right) .
\end{aligned}
$$

Hence they converge to unity as $T \rightarrow 0$, or $K=J / T \rightarrow \infty$ except for the terms $u_{l}^{L}$ and $v_{l}^{L}$. Using the scaling parameter $y=K / L$, we have the following asymptotic forms of $u_{l}^{L}$ and $v_{l}^{L}$ in the scaling limit: [5]

$$
\begin{aligned}
u_{l}(K)^{L} & =\left[1-\frac{l+1}{y} \frac{1}{L}+O\left(\frac{1}{L^{2}}\right)\right]^{L} \\
& \rightarrow \exp \left(-\frac{l+1}{y}\right), \\
v_{l}(K)^{L} & =\left[1-\frac{2 l+3}{y} \frac{1}{L}+O\left(\frac{1}{L^{2}}\right)\right]^{L} \\
& \rightarrow \exp \left(-\frac{2 l+3}{y}\right) .
\end{aligned}
$$

Further details are given in Appendix B.

In the thermodynamic limit $y \rightarrow 0$, the right-hand side of eq. (2.12) is reduced to

$$
\tilde{\chi}_{3}^{\text {peri }}(y \rightarrow 0) \rightarrow-\frac{44}{135}
$$

This value $-44 / 135$ is consistent with the value in the thermodynamic limit, eq. (1.11).

\section{B. Open boundary condition}

Next we consider the open system of length $L$. Because we handle only three modes $l=0,1,2$ of the modified Bessel functions in the open 
systems, it is easier to calculate the correlation functions in the open systems than in the periodic systems. The partition function is identical to unity. Fisher [7] gave the two-point function as follows:

$$
\left\langle n_{1}^{z} n_{1+M}^{z}\right\rangle^{\text {open }}=\frac{1}{3} u_{0}(K)^{M}
$$

Tomita and Mashiyama [8] gave the four-point function as follows:

$$
\left\langle n_{1}^{z} n_{1+A}^{z} n_{1+A+B}^{z} n_{1+A+B+C}^{z}\right\rangle^{\text {open }}=\frac{1}{9} u_{0}(K)^{A}\left[\frac{4}{5} v_{0}(K)^{B}+1\right] u_{0}(K)^{C} .
$$

Using these expressions, we already reported [5] the finite-size scaling function of the third-order susceptibility with the open boundary condition for the classical Heisenberg chain as follows:

$$
\begin{aligned}
\tilde{\chi}_{3}^{\text {open }}(y)= & -\frac{44}{135}-\frac{32}{45} \exp \left(-\frac{1}{y}\right)+\frac{2 y}{405}\left[1-\exp \left(-\frac{1}{y}\right)\right] \\
& \times\left[169+43 \exp \left(-\frac{1}{y}\right)-2 \exp \left(-\frac{2}{y}\right)\right] .
\end{aligned}
$$

In the thermodynamic limit $y \rightarrow 0$, we have the value of the finite-size scaling function of the third-order susceptibility as

$$
\tilde{\chi}_{3}^{\text {open }}(y \rightarrow 0) \rightarrow-\frac{44}{135} .
$$

This value is consistent with the eq. (1.11) as well as in the periodic case (2.17).

\section{FINITE-SIZE CALCULATION FOR $S=1 / 2$ AND $S=1$}

In the previous section, we obtained the finite-size scaling function $\tilde{\chi}_{3}$ for the classical systems with the periodic and open boundary conditions. We conjecture [3, 0 ] that this scaling function is common to the ferromagnetic Heisenberg chain with an arbitrary magnitude of the spin. 
In this section, in order to check this conjecture, we calculate the thirdorder susceptibility (2.2) of finite systems with $S=1 / 2$ and $S=1$ by the Householder method and compare the numerical data in the scaling limit with the finite-size scaling function (2.12) and (2.20).

\section{A. Periodic boundary condition}

First, we treat the periodic system. We diagonalized the Heisenberg Hamiltonian (1.1) numerically by the Householder method. The system size is up to $L=14$ for $S=1 / 2$ and up to $L=10$ for $S=1$. We then calculated the spin correlation functions and summed them up over all sites following eq. (2.2). We thus obtained the third-order nonlinear susceptibility numerically.

We plot the numerical data of $S=1 / 2$ chains with the periodic boundary condition in Fig. 3 and those of $S=1$ chain with the periodic boundary condition in Fig. 1 . We observe corrections to finite-size scaling in these figures. In order to take the scaling limit, or to exclude the corrections, we here assume that the leading correction is of the same form as in the linear susceptibility: [5]

$$
\chi_{3}(T, L)=\frac{J^{3}}{T^{6}}\left[\tilde{\chi}_{3}(y)+O(\sqrt{T / J})\right] .
$$

In the previous paper [5] we take the scaling limit, fitting the data quadratically with respect to $\sqrt{T / J}$. In the present analysis, however, the quadratic fitting resulted in spurious scaling limit. Some of the extrapolation curves for the quadratic fitting have the maximum around $\sqrt{T / J} \sim 0.2$ and start decreasing as $T / J \rightarrow 0$. This indicates that corrections higher than the second order are large in the nonlinear susceptibility. We therefore used corrections to finite-size scaling up to the third-order term, i.e. $(T / J)^{3 / 2}$. Each fitting curve in Figs. 3 and 10 was determined by the four points nearest to the ordinate. The crosses on the ordinate of Figs. 3 and 4 denote the scaling limit $\tilde{\chi}_{3}^{\text {peri }}(y)$.

In Fig. 5, we summarize the data in the scaling limit obtained in Figs. 3 and 4 together with the finite-size scaling function for the classical case 
(2.12). (In calculating eq. 2.12) we numerically summed the terms up to $l=100$, which turned out to be sufficient for the convergence of the series.) The data for $S=1 / 2$ and $S=1$ are quite consistent with the finite-size scaling function for $S=\infty$. This suggests that the finite-size scaling function (2.12), which is given analytically for the classical chain, does not depend on the magnitude of the spin $S$.

\section{B. Open boundary condition}

Next, we treat the open chain. We perform in Figs. 6 and 7 the same analysis as in the periodic systems, i.e. Figs. 3 and 4 . The finite-size scaling function (2.20) and the scaling-limit data from Figs. 6 and 7 are summarized in Fig. 8. We conclude that the finite-size scaling function (2.20) is common to all magnitudes of the spin for the open systems as well as for the periodic systems.

\section{DISCUSSION}

By using the numerical calculations for the quantum case, namely $S=1 / 2$ and $S=1$, we showed that the finite-size scaling function $\tilde{\chi}_{3}$ derived from the classical case is universal with respect to the magnitude of the spin both for the periodic and the open boundary conditions. The scaling function $\tilde{\chi}_{3}$ is exactly given by eq. (2.12) and eq. (2.20) for the periodic and the open chain, respectively. We emphasize that the universality both of the linear and the third-order susceptibilities implies the universality (1.5) of the magnetization of the arbitrary-spin ferromagnetic Heisenberg chain.

The universality of the scaling function implies that the spin-wave excitation in the present systems show universal behavior. The ground state of the arbitrary- $S$ Heisenberg ferromagnet in a magnetic field is

obviously the state where all the spins are aligned in the direction of the field. The thermal fluctuation creates spin-wave excitations. The present 
universality indicates that the thermally excited spin wave behaves independently of $S$. We can see this universal behavior as the universality of the correlation functions. We have already suggested [5] that the universality of the linear susceptibility originates in the universality of the two-point correlation function. We naturally expect that the universality of the nonlinear susceptibility originates in the universality of the two-point and the four-point correlation functions.

Indeed, we can derive the scaling forms of the correlation functions as follows. The finite-size scaling functions for the linear susceptibility and the nonlinear susceptibility are rederived from these scaling forms below. When the distance between two spins is large, we have the scaling function of the two-point correlation function for the periodic chain as follows: [5]

$$
\begin{aligned}
& \left\langle\left(\frac{S_{1}^{z}}{S}\right)\left(\frac{S_{1+M}^{z}}{S}\right)\right\rangle_{L}^{\text {peri }} \\
& \sim \frac{1}{\tilde{\mathcal{W}}} \sum_{l=0}^{\infty}\left\{(l+1) \exp \left[-\frac{l(l+1)}{2 y}\right]\right\} \\
& \quad \times\left[\exp \left(-\frac{M}{\xi_{l}}\right)+\exp \left(-\frac{L-M}{\xi_{l}}\right)\right]
\end{aligned}
$$

for ${ }^{\forall} S$ and $M,(L-M) \gg 1$, where the correlation length $\xi_{l}$ of the mode $l$ is defined by

$$
\xi_{l} \equiv \frac{K}{l+1}
$$

We also obtain from eq. (2.7) the scaling from of the four-point correlation function for the periodic chain as follows:

$$
\begin{aligned}
& \left\langle\left(\frac{S_{1}^{z}}{S}\right)\left(\frac{S_{1+A}^{z}}{S}\right)\left(\frac{S_{1+A+B}^{z}}{S}\right)\left(\frac{S_{1+A+B+C}^{z}}{S}\right)\right\rangle_{L}^{\text {peri }} \\
& \sim \frac{1}{\tilde{\mathcal{W}}} \sum_{l=0}^{\infty} \exp \left[-\frac{l(l+1)}{2 y}\right] \\
& \quad \times\left\{f _ { l } \left[\exp \left(-\frac{A}{\xi_{l}}\right) \exp \left(-\frac{B}{\xi_{l}}\right) \exp \left(-\frac{B}{\xi_{l+1}}\right) \exp \left(-\frac{C}{\xi_{l}}\right)\right.\right.
\end{aligned}
$$




$$
\begin{aligned}
& +\exp \left(-\frac{B}{\xi_{l}}\right) \exp \left(-\frac{C}{\xi_{l}}\right) \exp \left(-\frac{C}{\xi_{l+1}}\right) \exp \left(-\frac{D}{\xi_{l}}\right) \\
& +\exp \left(-\frac{C}{\xi_{l}}\right) \exp \left(-\frac{D}{\xi_{l}}\right) \exp \left(-\frac{D}{\xi_{l+1}}\right) \exp \left(-\frac{A}{\xi_{l}}\right) \\
& \left.+\exp \left(-\frac{D}{\xi_{l}}\right) \exp \left(-\frac{A}{\xi_{l}}\right) \exp \left(-\frac{A}{\xi_{l+1}}\right) \exp \left(-\frac{B}{\xi_{l}}\right)\right] \\
& \left.+g_{l}\left[\exp \left(-\frac{B}{\xi_{l}}\right) \exp \left(-\frac{D}{\xi_{l}}\right)+\exp \left(-\frac{A}{\xi_{l}}\right) \exp \left(-\frac{C}{\xi_{l}}\right)\right]\right\}
\end{aligned}
$$

for ${ }^{\forall} S$ and $A, B, C, D \gg 1$ with $A+B+C+D=L$.

For the open chain, we have obtained the scaling function of the twopoint correlation function as [0]

$$
\left\langle\left(\frac{S_{1}^{z}}{S}\right)\left(\frac{S_{1+M}^{z}}{S}\right)\right\rangle^{\text {open }}=\frac{1}{3} \exp \left(-M / \xi_{0}\right)
$$

for ${ }^{\forall} S$ and $M \gg 1$. We may also obtain the scaling function of the four-point correlation function as

$$
\begin{aligned}
& \left\langle\left(\frac{S_{1}^{z}}{S}\right)\left(\frac{S_{1+A}^{z}}{S}\right)\left(\frac{S_{1+A+B}^{z}}{S}\right)\left(\frac{S_{1+A+B+C}^{z}}{S}\right)\right\rangle^{\text {open }} \\
& \sim \frac{1}{9} \exp \left(-\frac{A}{\xi_{0}}\right)\left[\exp \left(-\frac{B}{\xi_{0}}\right) \exp \left(-\frac{B}{\xi_{1}}\right)+1\right] \exp \left(-\frac{C}{\xi_{0}}\right)
\end{aligned}
$$

for ${ }^{\forall} S$ and $A, B, C \gg 1$.

\section{ACKNOWLEDGMENTS}

The authors are grateful to Dr. Nishimori for providing the numerical package TITPACK version 2, and to Dr. Kaburagi and Dr. Tonegawa for the package KOBEPACK version 1.0. This work was supported in part by Grant-in-Aid for Scientific Research on Priority Areas, "Molecular Magnetism" (Area No 228) and "Infinite analysis" (Area No 231) ¿from the Ministry of Education, Science and Culture. Calculations which needed a large amount of the computer memory were done in the 
FACOM VPP500 of the Supercomputer Center, the Institute for Solid State Physics, the University of Tokyo.

\section{Appendix A:. FOUR-POINT CORRELATION FUNCTION OF THE CLASSICAL HEISENBERG CHAIN WITH THE PERIODIC BOUNDARY CONDITION}

In this Appendix we show the derivation of the four-point correlation function (2.7).

We consider the classical Heisenberg chain (2.1) with the periodic boundary condition. We choose the direction of the magnetic field $\mathbf{h}(|\mathbf{h}|=h)$ as the $z$ axis of the three-dimensional spin space. We define the polar coordinate $\theta_{i}$ and $\Phi_{i}$ of the $i$ th classical spin relative to the $z$ axis. We express each spin in the form

$$
\begin{aligned}
n_{i}^{z} & =\cos \theta_{i} \\
& =\sqrt{\frac{4 \pi}{3}} Y_{10}\left(\theta_{i}, \Phi_{i}\right) \\
& \equiv \sqrt{\frac{4 \pi}{3}} Y_{10}(i),
\end{aligned}
$$

where $Y_{l m}$ is the spherical harmonics. The exponential function of $\boldsymbol{n}_{i} \cdot \boldsymbol{n}_{i+1}$ is expanded in terms of the spherical harmonics as follows: [6]

$$
\begin{aligned}
\mathrm{e}^{K \boldsymbol{n}_{i} \boldsymbol{n}_{i+1}} & =\mathrm{e}^{K \cos \Theta} \\
& =4 \pi \sum_{l=0}^{\infty} \sum_{m=-l}^{l} \lambda_{l} Y_{l, m}(i) Y_{l, m}^{*}(i+1),
\end{aligned}
$$

where the parameter $\Theta$ is the angle between the spins $\boldsymbol{n}_{i}$ and $\boldsymbol{n}_{i+1}$, and $\lambda_{l}$ is defined by eq. (2.4).

In the following algebra, we use two formulas concerning the integration of the spherical harmonics. First, the integral of the product of two spherical harmonics over the solid angle $\Omega_{i}$ is given by [6] 


$$
\int_{0}^{2 \pi} \int_{0}^{\pi} Y_{l, m}^{*}(i) Y_{l^{\prime}, m^{\prime}}(i) \mathrm{d} \Omega_{i}=\delta_{l, l^{\prime}} \delta_{m, m^{\prime}},
$$

where $\delta$ is the Kronecker symbol. Next, the integral of the product of three spherical harmonics can be expressed in terms of Wigner's $3 n-j$ symbols: [6.9]

$$
\begin{aligned}
\int_{0}^{2 \pi} \mathrm{d} \Phi \int_{0}^{\pi} \sin \theta \mathrm{d} \theta Y_{l_{1}, m_{1}}^{*}(\theta, \Phi) Y_{l_{2}, m_{2}}(\theta, \Phi) Y_{l_{3}, m_{3}}(\theta, \Phi) \\
=(-1)^{m_{1}}\left[\frac{\left(2 l_{1}+1\right)\left(2 l_{2}+1\right)\left(2 l_{3}+1\right)}{4 \pi}\right]^{1 / 2} \\
\quad \times\left(\begin{array}{lll}
l_{1} & l_{2} & l_{3} \\
0 & 0 & 0
\end{array}\right)\left(\begin{array}{ccc}
l_{1} & l_{2} & l_{3} \\
-m_{1} & m_{2} & m_{3}
\end{array}\right)
\end{aligned}
$$

where Wigner's $3 n-j$ symbols can be written as follows:

$$
\begin{aligned}
& \left(\begin{array}{ccc}
l_{1} & l_{2} & l_{3} \\
m_{1} & m_{2} & m_{3}
\end{array}\right) \\
& \equiv \delta_{m_{1}+m_{2}+m_{3}, 0}\left[\frac{\left(l_{1}+l_{2}-l_{3}\right) !\left(l_{1}-l_{2}+l_{3}\right) !\left(-l_{1}+l_{2}+l_{3}\right) !}{\left(l_{1}+l_{2}+l_{3}+1\right) !}\right]^{\frac{1}{2}} \\
& \quad \times\left[\left(l_{1}+m_{1}\right) !\left(l_{1}-m_{1}\right) !\left(l_{2}+m_{2}\right) !\left(l_{2}-m_{2}\right) !\left(l_{3}+m_{3}\right) !\left(l_{3}-m_{3}\right) !\right]^{\frac{1}{2}} \\
& \quad \times \sum_{z} \frac{(-1)^{z+l_{1}-l_{2}-m_{3}}}{z !\left(l_{1}+l_{2}-l_{3}-z\right) !\left(l_{1}-m_{1}-z\right) !\left(l_{2}+m_{2}-z\right) !\left(l_{3}-l_{2}+m_{1}+z\right) !\left(l_{3}-l_{1}-m_{2}+z\right) !} .
\end{aligned}
$$

The summation in eq.(A.5) is over all integers $z$.

Using eqs. (A.1) - A.4), we can write down the four-point correlation function shown in Fig. 2 as

$$
\begin{aligned}
& F(A, B, C, D: K) \equiv Z_{L}\left\langle n_{1}^{z} n_{1+A}^{z} n_{1+A+B}^{z} n_{1+A+B+C}^{z}\right\rangle_{L}, \\
& =\left(\frac{3}{4 \pi}\right)^{2} \prod_{i=1}^{L} \int \frac{\mathrm{d} \Omega_{i}}{4 \pi} \exp \left(K \boldsymbol{n}_{i} \boldsymbol{n}_{i+1}\right) Y_{10}(1) Y_{10}(1+A)
\end{aligned}
$$




$$
\begin{aligned}
& \times Y_{10}(1+A+B) Y_{10}(1+A+B+C) \\
& =\left(\frac{3}{4 \pi}\right)^{2} \int \mathrm{d} \Omega_{1} \int \mathrm{d} \Omega_{1+A} \int \mathrm{d} \Omega_{1+A+B} \int \mathrm{d} \Omega_{1+A+B+C} \\
& \sum_{\left\{l_{1} m_{1}\right\}} \sum_{\left\{l_{2} m_{2}\right\}} \sum_{\left\{l_{3} m_{3}\right\}} \sum_{\left\{l_{4} m_{4}\right\}} \lambda_{l_{1}}^{A} \lambda_{l_{2}}^{B} \lambda_{l_{3}}^{C} \lambda_{l_{4}}^{D} \\
& \times Y_{l_{4} m_{4}}^{*}(1) Y_{10}(1) Y_{l_{1} m_{1}}(1) \times Y_{l_{1} m_{1}}^{*}(1+A) Y_{10}(1+A) Y_{l_{2} m_{2}}(1+A) \\
& \times Y_{l_{2} m_{2}}^{*}(1+A+B) Y_{10}(1+A+B) Y_{l_{3} m_{3}}(1+A+B) \\
& \times Y_{l_{3} m_{3}}^{*}(1+A+B+C) Y_{10}(1+A+B+C) Y_{l_{4} m_{4}}(1+A+B+C) \\
& =\left(\frac{3}{4 \pi}\right)^{2} \sum_{\left\{l_{1} m_{1}\right\}} \sum_{\left\{l_{2} m_{2}\right\}} \sum_{\left\{l_{3} m_{3}\right\}} \sum_{\left\{l_{4} m_{4}\right\}} \lambda_{l_{1}}^{A} \lambda_{l_{2}}^{B} \lambda_{l_{3}}^{C} \lambda_{l_{4}}^{D} \\
& \times(-1)^{m_{1}+m_{2}+m_{3}+m_{4}} \frac{9\left(2 l_{1}+1\right)\left(2 l_{2}+1\right)\left(2 l_{3}+1\right)\left(2 l_{4}+1\right)}{(4 \pi)^{2}} \\
& \times\left(\begin{array}{lll}
l_{4} & 1 & l_{1} \\
0 & 0 & 0
\end{array}\right)\left(\begin{array}{ccc}
l_{4} & 1 & l_{1} \\
-m_{4} & 0 & m_{1}
\end{array}\right)\left(\begin{array}{lll}
l_{1} & 1 & l_{2} \\
0 & 0 & 0
\end{array}\right)\left(\begin{array}{ccc}
l_{1} & 1 & l_{2} \\
-m_{1} & 0 & m_{2}
\end{array}\right) \\
& \times\left(\begin{array}{lll}
l_{2} & 1 & l_{3} \\
0 & 0 & 0
\end{array}\right)\left(\begin{array}{ccc}
l_{2} & 1 & l_{3} \\
-m_{2} & 0 & m_{3}
\end{array}\right)\left(\begin{array}{lll}
l_{3} & 1 & l_{4} \\
0 & 0 & 0
\end{array}\right)\left(\begin{array}{ccc}
l_{3} & 1 & l_{4} \\
-m_{3} & 0 & m_{4}
\end{array}\right) \text {. }
\end{aligned}
$$

In order to calculate eq. (A.7) further, we need the following formula for the Wigner symbol: 9]

$$
\left(\begin{array}{ccc}
l & 1 & l^{\prime} \\
-m & 0 & m^{\prime}
\end{array}\right)=\delta_{m, m^{\prime}}\left(\begin{array}{ccc}
l & 1 & l^{\prime} \\
-m & 0 & m
\end{array}\right)
$$

with

$$
\begin{aligned}
& \left(\begin{array}{ccc}
l & 1 & l^{\prime} \\
-m & 0 & m
\end{array}\right)=\delta_{l^{\prime}, l+1}\left(\begin{array}{ccc}
l & 1 & l+1 \\
-m & 0 & m
\end{array}\right)
\end{aligned}
$$

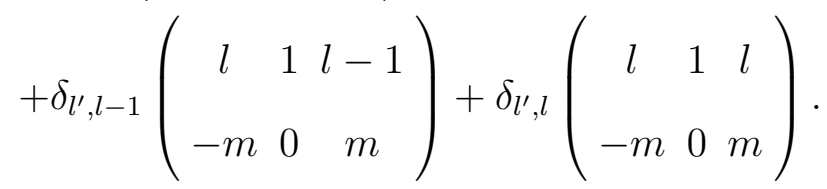


Each term in eq. (A.9) is given as follows: [9]

$$
\begin{aligned}
\left(\begin{array}{ccc}
l & 1 & l \\
-m & 0 & m
\end{array}\right) & =(-1)^{-l+m} \frac{m}{\sqrt{l(l+1)(2 l+3)}}, \\
\left(\begin{array}{ccc}
l & 1 & l+1 \\
-m & 0 & m
\end{array}\right) & =(-1)^{l-m+1} \sqrt{\frac{(l-m+1)(l+m+1)}{(l+1)(2 l+1)(2 l+3)}}, \\
\left(\begin{array}{ccc}
l & 1 & l-1 \\
-m & 0 & m
\end{array}\right) & =(-1)^{l-m} \sqrt{\frac{(l-m)(l+m)}{l(2 l-1)(2 l+1)}} .
\end{aligned}
$$

We substitute the $3 n-j$ symbols in eq. (A.7) with eqs. (A.8)-(A.12). Then we have the form

$$
\begin{aligned}
F & (A, B, C, D: K) \\
= & \sum_{\left\{l_{1} m_{1}\right\}} \sum_{\left\{l_{2} m_{2}\right\}} \sum_{\left\{l_{3} m_{3}\right\}} \sum_{\left\{l_{4} m_{4}\right\}} \lambda_{l_{1}}^{A} \lambda_{l_{2}}^{B} \lambda_{l_{3}}^{C} \lambda_{l_{4}}^{D} \\
& \times\left(2 l_{1}+1\right)\left(2 l_{2}+1\right)\left(2 l_{3}+1\right)\left(2 l_{4}+1\right) \\
& \times\left\{a\left(l_{4}+1, m\right) \delta_{l_{1}, l_{4}+1}+a\left(l_{4}, m\right) \delta_{l_{1}, l_{4}-1}\right\} \\
& \times\left\{a\left(l_{1}+1, m\right) \delta_{l_{2}, l_{1}+1}+a\left(l_{1}, m\right) \delta_{l_{2}, l_{1}-1}\right\} \\
& \times\left\{a\left(l_{2}+1, m\right) \delta_{l_{3}, l_{2}+1}+a\left(l_{2}, m\right) \delta_{l_{3}, l_{2}-1}\right\} \\
& \times\left\{a\left(l_{3}+1, m\right) \delta_{l_{4}, l_{3}+1}+a\left(l_{3}, m\right), \delta_{l_{4}, l_{3}-1}\right\}
\end{aligned}
$$

where

$$
a(l, m) \equiv(-1)^{m} \frac{\sqrt{l^{2}-m^{2}}}{(2 l-1)(2 l+1)} .
$$

Because of Kronecker's deltas, the nonvanishing contributions of the fourfold summation in eq. (A.13) come from the following six combinations: 


$$
\begin{aligned}
& \boldsymbol{l}_{\alpha 1} \equiv\left\{l_{1}=l, l_{2}=l+1, l_{3}=l+2, l_{4}=l+1\right\}, \\
& \boldsymbol{l}_{\alpha 2} \equiv\{l+1, l, l+1, l+2\}, \\
& \boldsymbol{l}_{\alpha 3} \equiv\{l+2, l+1, l, l+1\}, \\
& \boldsymbol{l}_{\alpha 4} \equiv\{l+1, l+2, l+1, l\}, \\
& \boldsymbol{l}_{\beta 1} \equiv\{l, l+1, l, l+1\}, \\
& \boldsymbol{l}_{\beta 2} \equiv\{l+1, l, l+1, l\} .
\end{aligned}
$$

Hence, it is sufficient to sum up the terms over the one parameter $l$. The summation of the contributions from the first four combinations, $\boldsymbol{l}_{\alpha 1}, \boldsymbol{l}_{\alpha 2}, \boldsymbol{l}_{\alpha 3}, \boldsymbol{l}_{\alpha 4}$, and that from the last two combinations, $\boldsymbol{l}_{\beta 1}, \boldsymbol{l}_{\beta 2}$, are given as follows, respectively:

$$
\begin{aligned}
F^{\alpha}(A, B, C, D: K) \equiv & \sum_{l, m}(2 l+1)(2 l+3)^{2}(2 l+5) \\
& \times a(l+1, m)^{2} a(l+2, m)^{2} \\
& \times\left(\lambda_{l}^{A} \lambda_{l+1}^{B} \lambda_{l+2}^{C} \lambda_{l+1}^{D}+\lambda_{l}^{B} \lambda_{l+1}^{C} \lambda_{l+2}^{D} \lambda_{l+1}^{A}\right. \\
& \left.+\lambda_{l}^{C} \lambda_{l+1}^{D} \lambda_{l+2}^{A} \lambda_{l+1}^{B}+\lambda_{l}^{D} \lambda_{l+1}^{A} \lambda_{l+2}^{B} \lambda_{l+1}^{C}\right) \\
= & \sum_{l=0}^{\infty} f_{l} \lambda_{l}^{L}\left[u_{l}^{B} v_{l}^{C} u_{l}^{D}+u_{l}^{C} v_{l}^{D} u_{l}^{A}\right. \\
& \left.+u_{l}{ }^{D} v_{l}^{A} u_{l}^{B}+u_{l}^{A} v_{l}^{B} u_{l}^{C}\right],
\end{aligned}
$$

and

$$
\begin{aligned}
F^{\beta}(A, B, C, D: K) \equiv & \sum_{l, m}(2 l+1)^{2}(2 l+3)^{2} a(l+1, m)^{4} \\
& \times\left[\lambda_{l}^{A} \lambda_{l+1}^{B} \lambda_{l}^{C} \lambda_{l+1}^{D}+\lambda_{l+1}^{A} \lambda_{l}^{B} \lambda_{l+1}^{C} \lambda_{l}^{D}\right] \\
= & \sum_{l=0}^{\infty} g_{l} \lambda_{l}^{L}\left[u_{l}^{B} u_{l}^{D}+u_{l}^{A} u_{l}^{C}\right] .
\end{aligned}
$$

Here the functions $u_{l}$ and $v_{l}$, and the coefficient $f_{l}$ and $g_{l}$ are defined by eqs. (2.6), (2.10), (2.8) and (2.9), respectively.

Summing up (A.21) and (A.22), we arrive at the analytic expression of the four-point correlation function in the form eq. (2.7). 


\section{Appendix B:. THE THIRD-ORDER NONLINEAR}

\section{SUSCEPTIBILITY OF THE CLASSICAL HEISENBERG \\ CHAIN WITH THE PERIODIC BOUNDARY CONDITION}

We write the finite-size scaling function of the third-order nonlinear susceptibility for the periodic system as eq. (2.12). We present a deviation of this finite-size scaling function in this Appendix.

We substitute the correlation functions in the right-hand side of eq. (2.2) with eqs. (2.5) and (2.7). We divide this summation into the summation of the four-point correlation functions $\Phi$ and that of the two-point correlation functions $\Psi$ as follows:

$$
\chi_{3}^{\text {peri }}(T, L)=\frac{1}{3 ! L T^{3}}[\Phi(T, L)-\Psi(T, L)],
$$

where

$$
\begin{aligned}
\Phi(T, L) & \equiv \sum_{i_{1}, i_{2}, i_{3}, i_{4}}\left\langle n_{i_{1}}^{z} n_{i_{2}}^{z} n_{i_{3}}^{z} n_{i_{4}}^{z}\right\rangle_{L}^{\text {peri }}, \\
\Psi(T, L) & \equiv 3\left[\sum_{i_{1}, i_{2}}\left\langle n_{i_{1}}^{z} n_{i_{2}}^{z}\right\rangle_{L}^{\text {peri }}\right]^{2} .
\end{aligned}
$$

Let us show in the following the calculations of $\Phi$ in details. The fourfold summation over the sites $i_{1}, i_{2}, i_{3}$ and $i_{4}$ in eq. (B.2) is reduced to the threefold summation because of the translational invariance of the periodic system. By using the parameters $A, B, C$ and $D$ shown in Fig. 2, we express the summation of the four-point correlation function, $\Phi$, as follows:

$$
\begin{aligned}
\Phi(T, L)= & \frac{L}{Z_{L}}\left[\phi_{1}(T, L)+\phi_{2}(T, L)+\phi_{3}(T, L)+\phi_{4}(T, L)\right. \\
& +F(0,0,0, L: K)]
\end{aligned}
$$

where $Z_{L}$ is the partition function (2.3), $F$ is defined by eq. (A.6), and

$$
\phi_{1}(T, L) \equiv(4-1) ! \sum_{1 \leq A, B, C, D \leq L} \delta_{L, A+B+C+D} F(A, B, C, D: K)
$$




$$
\begin{aligned}
& \phi_{2}(T, L) \equiv 12 \sum_{1 \leq B, C, D \leq L} \delta_{L, B+C+D} F(0, B, C, D: K), \\
& \phi_{3}(T, L) \equiv 4 \sum_{1 \leq C, D \leq L} \delta_{L, C+D} F(0,0, C, D: K) \\
& \phi_{4}(T, L) \equiv{ }_{3} \mathrm{C}_{1} \sum_{1 \leq C, D \leq L} \delta_{L, C+D} F(0, C, 0, D: K) .
\end{aligned}
$$

Now we calculate each term of (B.4) as follows. First, using eq. (2.7), we write $\phi_{1}$ explicitly in the form

$$
\begin{aligned}
\frac{1}{6} \phi_{1}(T, L)= & \sum_{A, B, C, D} \delta_{L, A+B+C+D} \\
& \times \sum_{l=0}^{\infty} \lambda_{l}^{L}\left\{f_{l}\left[u_{l}^{A} v_{l}^{B} u_{l}^{C}+u_{l}^{B} v_{l}^{C} u_{l}^{D}+u_{l}^{C} v_{l}^{D} u_{l}^{A}+u_{l}^{D} v_{l}^{A} u_{l}^{B}\right]\right. \\
& \left.+g_{l}\left[u_{l}^{A} u_{l}^{C}+u_{l}^{B} u_{l}^{D}\right]\right\} \\
= & \sum_{l} \lambda_{l}^{L}\left\{4 f_{l} V_{1}(K, L)+2 g_{l} V_{2}(K, L)\right\}
\end{aligned}
$$

where we define $V_{1}$ and $V_{2}$ as

$$
\begin{aligned}
& V_{1}(K, L) \equiv \sum_{A=1}^{L-3} \sum_{B=1}^{L-A-2} \sum_{C=1}^{L-A-B-1} u_{l}(K)^{A} v_{l}(K)^{B} u_{l}(K)^{C} \\
& V_{2}(K, L) \equiv \sum_{A=1}^{L-3} \sum_{B=1}^{L-A-2} \sum_{C=1}^{L-A-B-1} u_{l}(K)^{A} u_{l}(K)^{C}
\end{aligned}
$$

In obtaining the final expression of $(\mathbb{B} .9)$, we use the invariance of the summation of $u_{l}^{A} v_{l}^{B} u_{l}^{C}$ and $u_{l}^{A} u_{l}^{C}$ under the cyclic permutation among $A, B, C$ and $D$. Each summation in $(\mathbb{B} .10)$ and $(\mathbb{B} .11)$ can be carried out in the form

$$
\sum_{n=1}^{N} x^{n}=\left(1-x^{N}\right) E(x)
$$

with 


$$
E(x) \equiv \frac{x}{1-x},
$$

for $|x|<1$. We thereby obtain $V_{1}$ as follows:

$$
\begin{aligned}
V_{1}= & E\left(u_{l}\right) \sum_{A=1}^{L-3} \sum_{B=1}^{L-A-2}\left[u_{l}^{A} v_{l}^{B}-u_{l}^{L-1} u_{l+1}^{B}\right] \\
= & E\left(u_{l}\right) \sum_{A=1}^{L-3}\left\{E\left(v_{l}\right)\left[u_{l}^{A}-v_{l}^{L-2}\left(\frac{1}{u_{l+1}}\right)^{A}\right]-u_{l}^{L-1} E\left(u_{l+1}\right)\left[1-u_{l+1}^{L-2-A}\right]\right\} \\
= & E\left(u_{l}\right)\left\{-E\left(u_{l+1}\right) u_{l}^{L-1}(L-3)+E\left(u_{l}\right) E\left(v_{l}\right)\left(1-u_{l}^{L-3}\right)\right. \\
& \left.-\left[E\left(v_{l}\right)-u_{l} E\left(u_{l+1}\right)\right] E\left(\frac{1}{u_{l+1}}\right) v_{l}\left(v_{l}^{L-3}-u_{l}^{L-3}\right)\right\} .
\end{aligned}
$$

Here we have used the relation $v_{l} / u_{l}=u_{l+1}$; see the definitions (2.6) and (2.10). In a similar way we have $V_{2}$ as

$$
\begin{aligned}
V_{2}= & E\left(u_{l}\right) \sum_{A=1}^{L-3} \sum_{B=1}^{L-A-2} u_{l}^{A}\left(1-u_{l}^{L-1-A-B}\right) \\
= & E\left(u_{l}\right) \sum_{A=1}^{L-3}\left[u_{l}^{A}(L-2-A)+\frac{1}{1-u_{l}}\left(u_{l}^{L-1}-u_{l}^{A+1}\right)\right] \\
= & E\left(u_{l}\right)^{2}\left\{\left(1+u_{l}\right)(L-3) u_{l}^{L-3}\right. \\
& \left.+\left(1-u_{l}^{L-3}\right)\left[L-2-\frac{E\left(u_{l}\right)}{u_{l}}-E\left(u_{l}\right)\right]\right\} .
\end{aligned}
$$

The calculations of $\phi_{2}, \phi_{3}$ and $\phi_{4}$ are done in the same way. By using eq. (2.7), we can write down the function $\phi_{2}$ in the form

$$
\begin{aligned}
\frac{1}{12} \phi_{2}(T, L)= & \sum_{l=0}^{\infty} \lambda_{l}^{L}\left\{f_{l}\left[V_{3}(K, L)+2 V_{4}(K, L)+V_{5}(K, L)\right]\right. \\
& \left.+g_{l}\left[V_{6}(K, L)+V_{5}(K, L)\right]\right\}
\end{aligned}
$$

where we have

$$
\begin{aligned}
V_{3}(K, L) & \equiv \sum_{C=1}^{L-2} \sum_{B=1}^{L-1-C} u_{l}^{B} v_{l}^{C} u_{l}^{L-B-C} \\
& =E\left(u_{l+1}\right)\left[(L-2) u_{l}^{L}-u_{l}^{2} E\left(u_{l+1}\right)\left(u_{l}^{L-2}-v_{l}^{L-2}\right)\right]
\end{aligned}
$$




$$
\begin{aligned}
& V_{4}(K, L) \equiv \sum_{C=1}^{L-2} \sum_{B=1}^{L-1-C} v_{l}^{B} u_{l}^{C} \\
&=E\left(v_{l}\right)\left[E\left(u_{l}\right)\left(1-u_{l}^{L-2}\right)-v_{l} E\left(\frac{1}{u_{l+1}}\right)\left(v_{l}^{L-2}-u_{l}^{L-2}\right)\right] \\
& V_{5}(K, L) \equiv \sum_{C=1}^{L-2} \sum_{B=1}^{L-1-C} u_{l}^{B} u_{l}^{C} \\
&=E\left(u_{l}\right)\left[E\left(u_{l}\right)\left(1-u_{l}^{L-2}\right)-(L-2) u_{l}^{L-1}\right]
\end{aligned}
$$

and

$$
\begin{aligned}
V_{6}(K, L) & \equiv \sum_{C=1}^{L-2} \sum_{B=1}^{L-1-C} u_{l}^{B} \\
& =E\left(u_{l}\right)\left[L-2-E\left(u_{l}\right)\left(1-u_{l}^{L-2}\right)\right] .
\end{aligned}
$$

The function $\phi_{3}$ is given by

$$
\frac{1}{4} \phi_{3}(T, L)=2 \sum_{l=0}^{\infty} \lambda_{l}^{L}\left\{f_{l}\left[V_{7}(K, L)+V_{8}(K, L)\right]+g_{l} V_{8}(K, L)\right\}
$$

where

$$
V_{7}(K, L) \equiv \sum_{C=1}^{L-1} v_{l}^{C} u_{l}^{L-C}=u_{l} E\left(u_{l+1}\right)\left(u_{l}^{L-1}-v_{l}^{L-1}\right),
$$

and

$$
V_{8}(K, L) \equiv \sum_{C=1}^{L-1} u_{l}^{C}=E\left(u_{l}\right)\left(1-u_{l}^{L-1}\right)
$$

The function $\phi_{4}$ is written as

$$
\begin{aligned}
\frac{1}{3} \phi_{4}(T, L)= & \sum_{l=0}^{\infty} \lambda_{l}^{L}\left\{2 f_{l}\left[(L-1) u_{l}^{L}+E\left(v_{l}\right)\left(1-v_{l}^{L-1}\right)\right]\right. \\
& \left.+g_{l}\left(1+u_{l}^{L}\right)(L-1)\right\} .
\end{aligned}
$$


We thereby have the expression of the function $\Phi$ in (B.2).

We can calculate the function $\Psi$ in $(\mathrm{B} .3$ much simpler. Using eq. (2.5), we obtain the explicit form of $\Psi$ as follows:

$$
\begin{aligned}
\frac{3 Z_{L}^{2}}{L^{2}} \Psi(T, L) & =\left\{\sum_{l=0}^{\infty}(l+1) \lambda_{l}^{L}\left[1+u_{l}^{L}+\sum_{M=1}^{L-1}\left(u_{l}^{M}+u_{l}^{L-M}\right)\right]\right\}^{2} \\
& =\left\{\sum_{l=0}^{\infty}(l+1) \lambda_{l}^{L}\left[1+u_{l}^{L}+2 E\left(u_{l}\right)\left(1-u_{l}^{L-1}\right)\right]\right\}^{2} .
\end{aligned}
$$

The above expressions (B.2)-(B.25) give a final expression of the thirdorder nonlinear susceptibility. The expressions in the thermodynamic limit are given in ref. 3 .

Finally, let us explain the calculation of the scaling limit of the nonlinear susceptibility, namely eq. (2.12). The scaling limit is the limit $K \rightarrow \infty$ with $y=K / L$ fixed. It is important to notice that the functions $u_{l}$ and $v_{l}$ converge to unity in the scaling limit, except for the terms $u_{l}^{L}$ and $v_{l}^{L}$, which turn out to be exponential functions; see eqs. (2.13)-(2.16).

The functions $E\left(u_{l}\right)$ and $E\left(v_{l}\right)$, on the other hand, behave as follows at low temperatures:

$$
\begin{gathered}
E\left(u_{l}\right)=\frac{u_{l}}{1-u_{l}} \simeq \frac{K}{l+1}, \\
E\left(\frac{1}{u_{l}}\right)=\frac{\frac{1}{u_{l}}}{1-\frac{1}{u_{l}}} \simeq-\frac{K}{l+1}, \\
E\left(v_{l}\right)=\frac{v_{l}}{1-u_{l}} \simeq \frac{K}{2 l+3} .
\end{gathered}
$$

These terms partly contribute to the prefactor $J^{3} / T^{6}$ of $\chi_{3}$ in (1.9). Since we factored out $T^{-3}$ from $\chi_{3}$ in the expression (B.1), we pick out the terms of the order of $J^{3} / T^{3}=K^{3}$ from the functions $\Phi$ and $\Psi$. (The factor $1 / L$ in (B.1) is cancelled out by the factor $L$ in (B.4).) For example, the function $V_{1}$ in (B.14) is reduced in the scaling limit to the following form:

$$
V_{1} \simeq \frac{K}{l+1}\left\{-\frac{K}{l+2} \frac{K}{y} \mathrm{e}^{-(l+1) / y}+\frac{K}{l+1} \frac{K}{2 l+3}\left(1-\mathrm{e}^{-(l+1) / y}\right)\right.
$$




$$
\left.+\left(\frac{K}{2 l+3}-\frac{K}{l+2}\right) \frac{K}{l+2}\left(\mathrm{e}^{-(2 l+3) / y}-\mathrm{e}^{-(l+1) / y}\right)\right\} .
$$

It is notable that the functions $\phi_{2}, \phi_{3}$ and $\phi_{4}$ yield terms only of lower orders of $K$ than $K^{3}$, and hence vanish in the scaling limit. Thereby we arrive at eq. (2.12). 
[1] F. D. M. Haldane: Phys. Lett. 93 A (1983) 464.

[2] F. D. M. Haldane: Phys. Rev. Lett. 50 (1983) 1153.

[3] H. Nakamura and M. Takahashi: J. Phys. Soc. Jpn. 63 (1994) 2563.

[4] M. Takahashi: Phys. Rev. B 44 (1991) 12382.

[5] H. Nakamura N. Hatano and M. Takahashi: J. Phys. Soc. Jpn. 64 (1995) 1955.

[6] G.S. Joyce: Phys. Rev. 155 (1967) 478.

[7] M.E. Fisher: Am. J. Phys. 32 (1964) 343.

[8] H. Tomita and M. Mashiyama: Prog. Theor. Phys. 48 (1972) 1133.

[9] L. D. Landau and E. M. Lifshitz: Quantum Mechanics (Pergamon Press, New York, 1977) 3rd ed., Vol.3 of Course of Theoretical Physics, Chap. XIV, p. 431. 


\section{Figure Captions}

1. The spin configuration for the two-point correlation function of Heisenberg chain with the periodic boundary condition. Total number of spin is $L$.

2. The spin configuration for the four-point correlation function of Heisenberg chain with the periodic boundary condition. Total number of spin is $L$.

3. The correction to finite-size scaling of $\chi_{3} T^{6} / J^{3}$ for $y=J /(T L)=$ $1.0,1.2,1.4,1.6,1.8,2.0$ in the case of the $S=1 / 2$ ferromagnetic Heisenberg chain with the periodic boundary condition. We used systems with $L$ up to 14 . We assumed that the leading correction is of the form $\sqrt{T / J}$. Each solid line indicates the extrapolating function based on the four points nearest to the ordinate.

4. The correction to finite-size scaling of $\chi_{3} T^{6} / J^{3}$ for $y=J /(T L)=$ $1.0,1.2,1.4,1.6,1.8,2.0$ in the case of the $S=1$ ferromagnetic Heisenberg chain with the periodic boundary condition. We used systems with $L$ up to 10 . We assumed that the leading correction is of the form $\sqrt{T / J}$. Each solid line indicates the extrapolating function based on the four points nearest to the ordinate.

5. The finite-size scaling function $\tilde{\chi}_{3}{ }^{\text {peri }}(y)$ of the ferromagnetic Heisenberg chain with the periodic boundary condition, (2.12). The solid curve indicates the scaling function $\tilde{\chi}_{3}^{\text {peri }}$ analytically obtained for $S=\infty$. The numerical data, which are extrapolated in Figs. 3 and 4 , are indicated by circles for $S=1 / 2$ and by crosses for $S=1$. We can obtain the value in the thermodynamic limit, $\tilde{\chi}_{3}{ }^{\text {peri }}(y=0)$ for $S=1 / 2$, by the Bethe-Ansatz method as in (1.11).

6. The correction to finite-size scaling of $\chi_{3} T^{6} / J^{3}$ for $y=J /(T L)$ $=0.6,0.8,1.0,1.2,2.0$ in the case of the $S=1 / 2$ ferromagnetic Heisenberg chain with the open boundary condition. We used systems with $L$ up to 14 . We assumed that the leading correction 
is of the form $\sqrt{T / J}$. Each solid line indicates the extrapolating function based on the four points nearest to the ordinate.

7. The correction to finite-size scaling of $\chi_{3} T^{6} / J^{3}$ for $y=J /(T L)=$ $0.6,0.8,1.0,1.2,2.0$ in the case of the $S=1$ ferromagnetic Heisenberg chain with the open boundary condition. We used systems with $L$ up to 10 . We assumed that the leading correction is of the form $\sqrt{T / J}$. Each solid line indicates the extrapolating function based on the four points nearest to the ordinate.

8. The finite-size scaling function $\tilde{\chi}_{1}{ }^{\text {open }}(y)$ of the ferromagnetic Heisenberg chain with the open boundary condition, (2.20). The solid curve indicates the scaling function $\tilde{\chi}_{3}^{\text {open }}$ analytically obtained for $S=\infty$. The numerical data, which are extrapolated in Figs. 6 and 7 , are indicated by circles for $S=1 / 2$ and by crosses for $S=1$. 


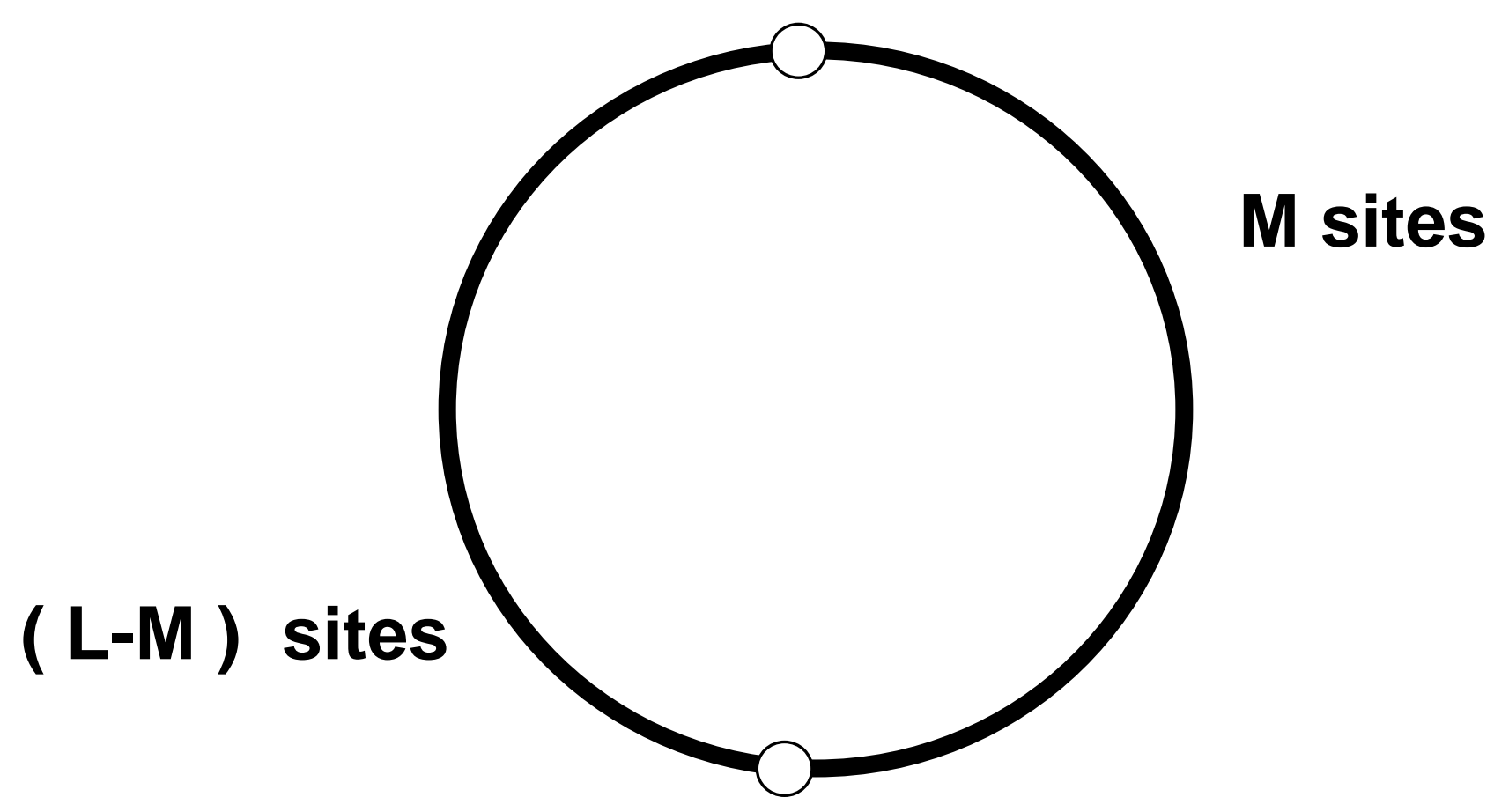

Fig.1 H.Nakamura, N. Hatano \& M. Takahashi 


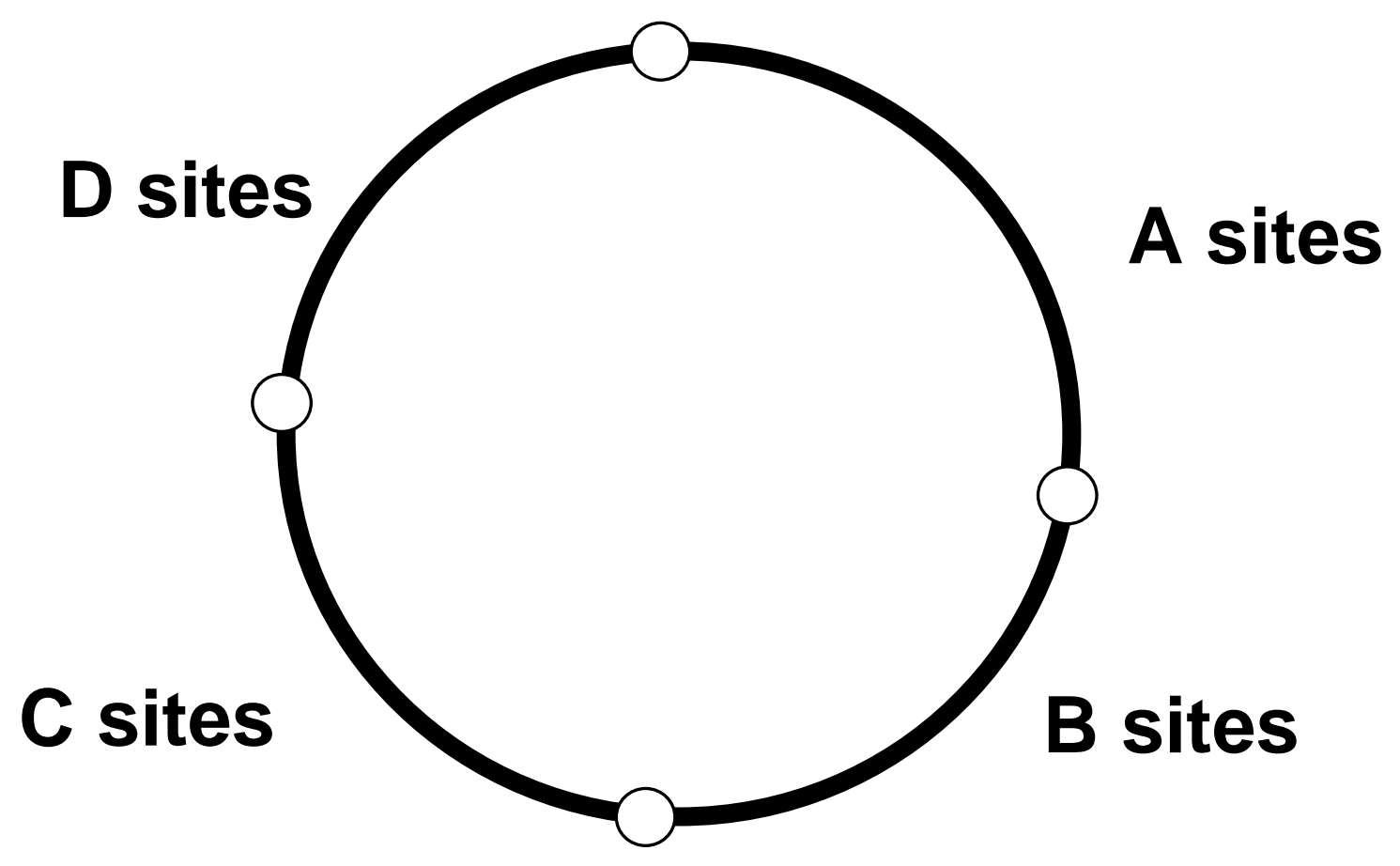

$$
L=A+B+C+D
$$

Fig.2. H.Nakamura, N. Hatano \& M. Takahashi 


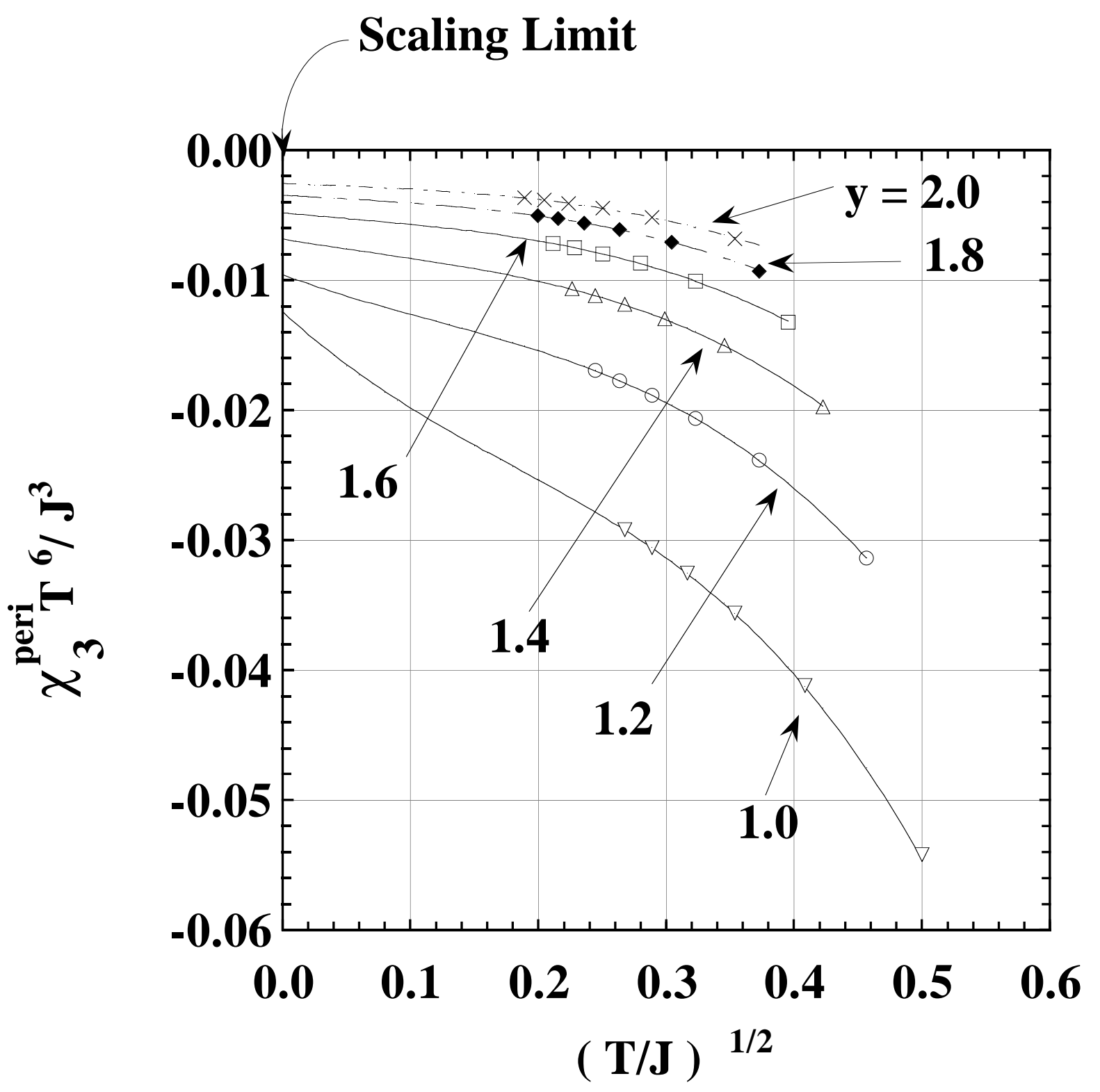

Fig.3. H. Nakamura, N. Hatano \& M. Takahashi 


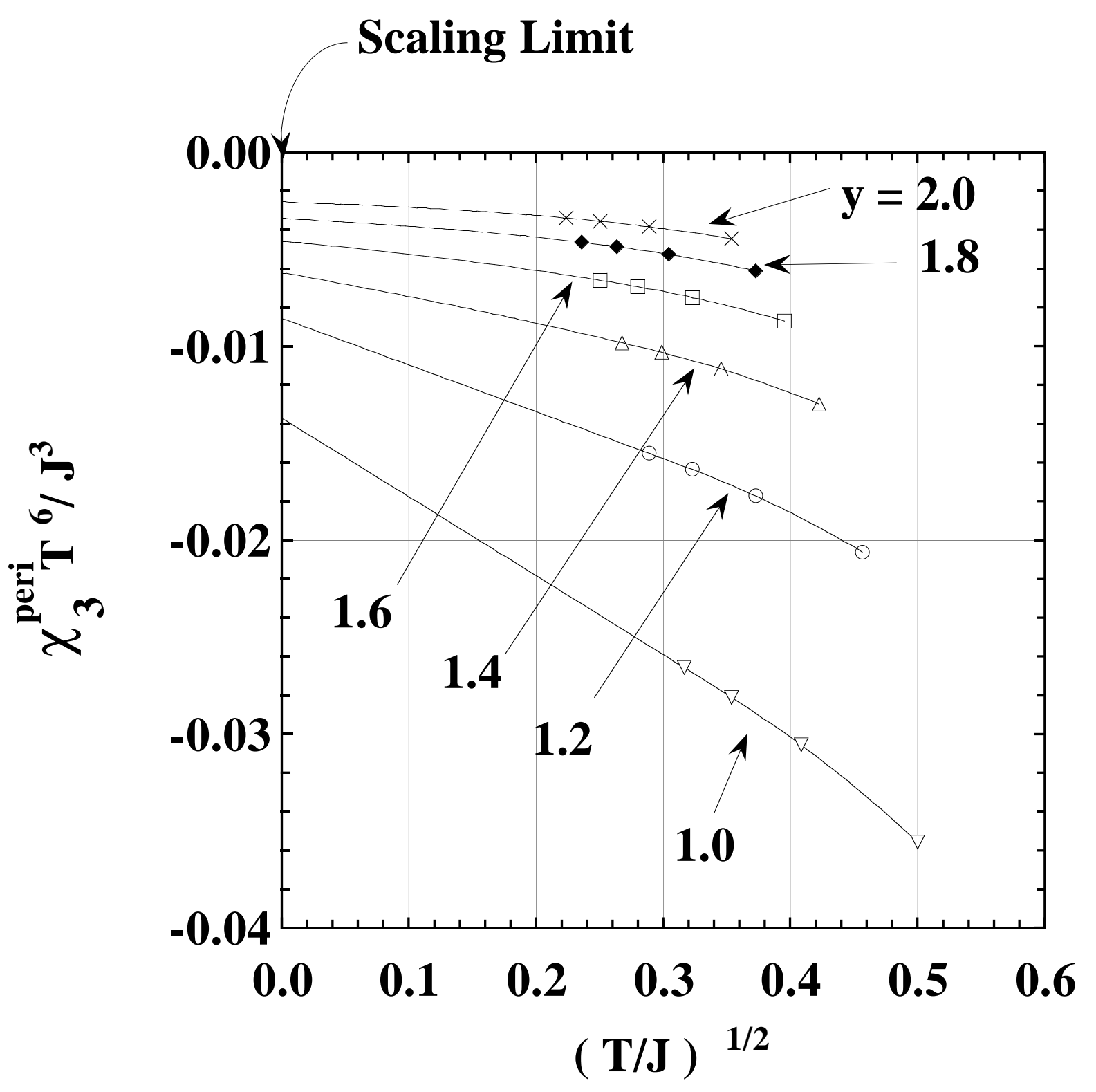

Fig.4. H. Nakamura, N. Hatano \& M. Takahashi 


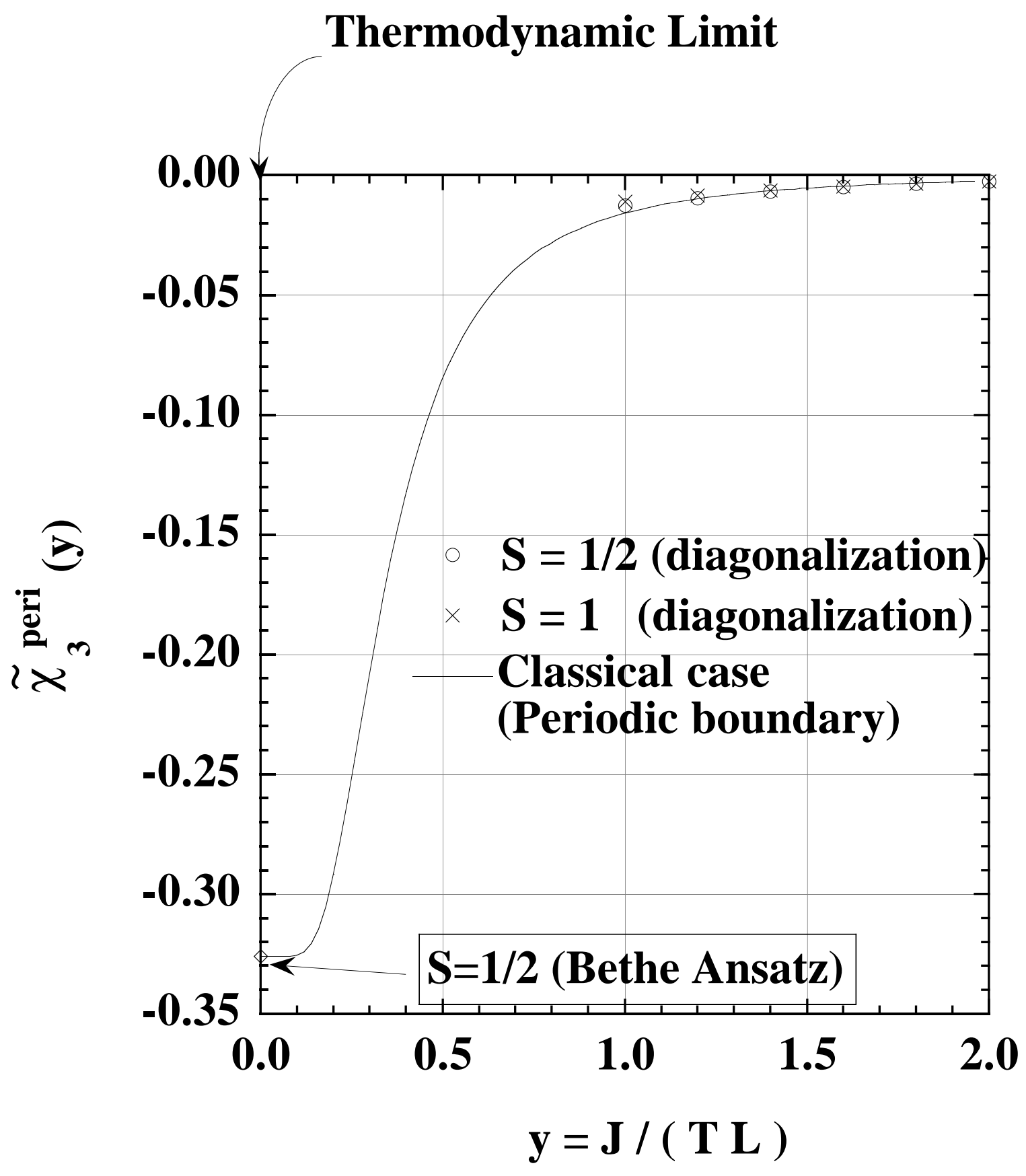

Fig.5. H. Nakamura, N. Hatano \& M. Takahashi 


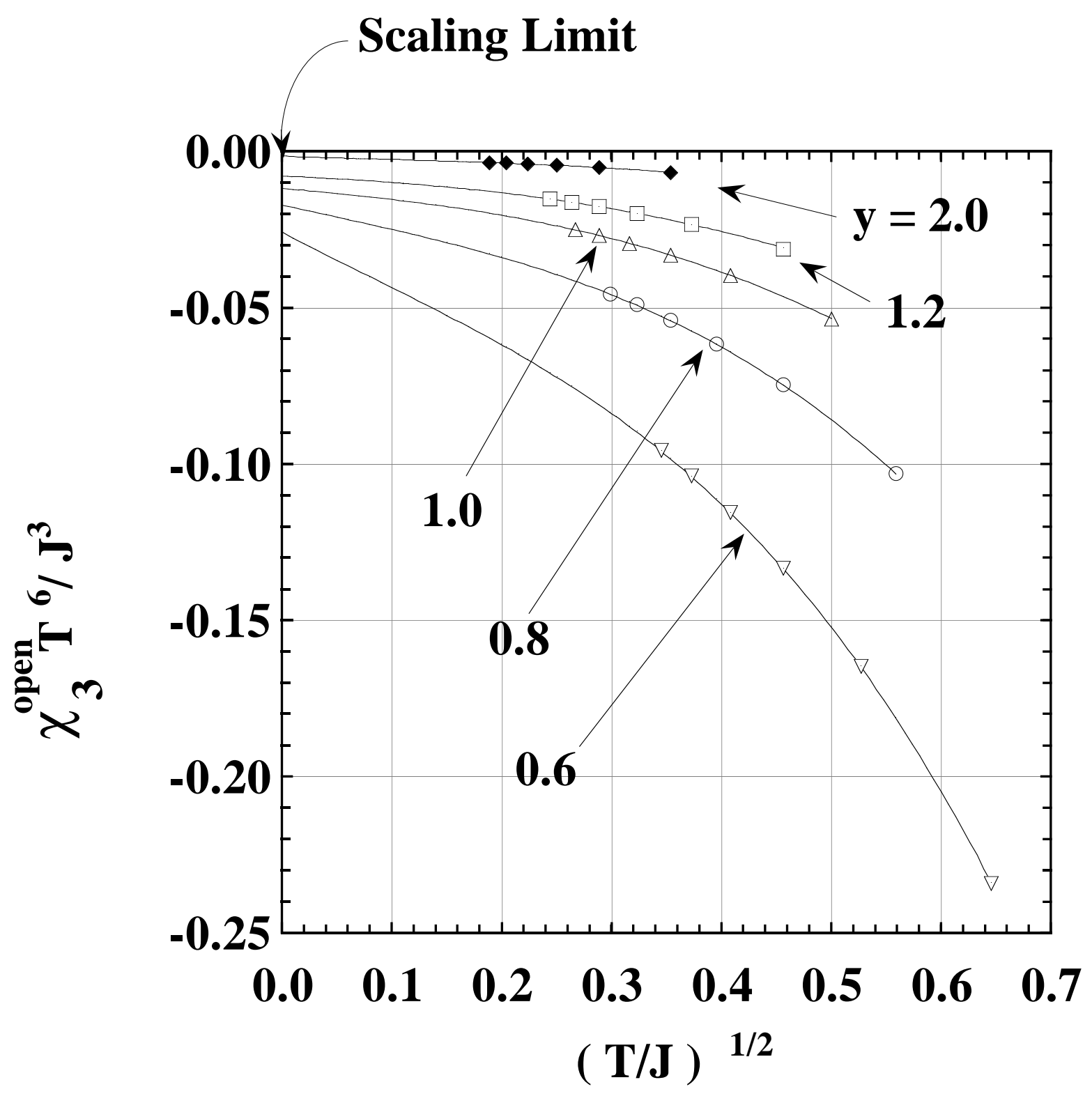

Fig.6. H. Nakamura, N. Hatano \& M. Takahashi 


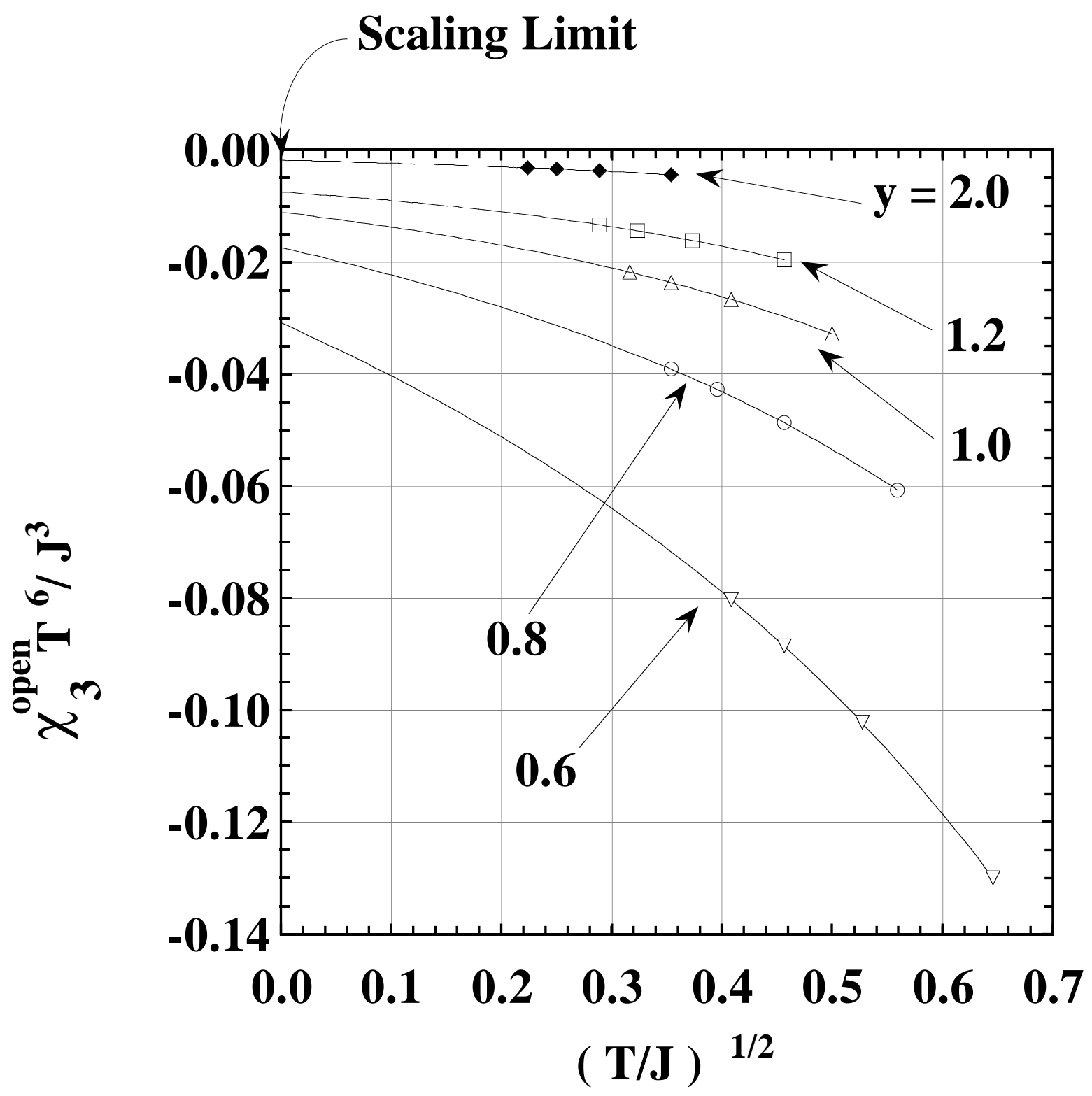

Fig.7. H. Nakamura, N. Hatano \& M. Takahashi 


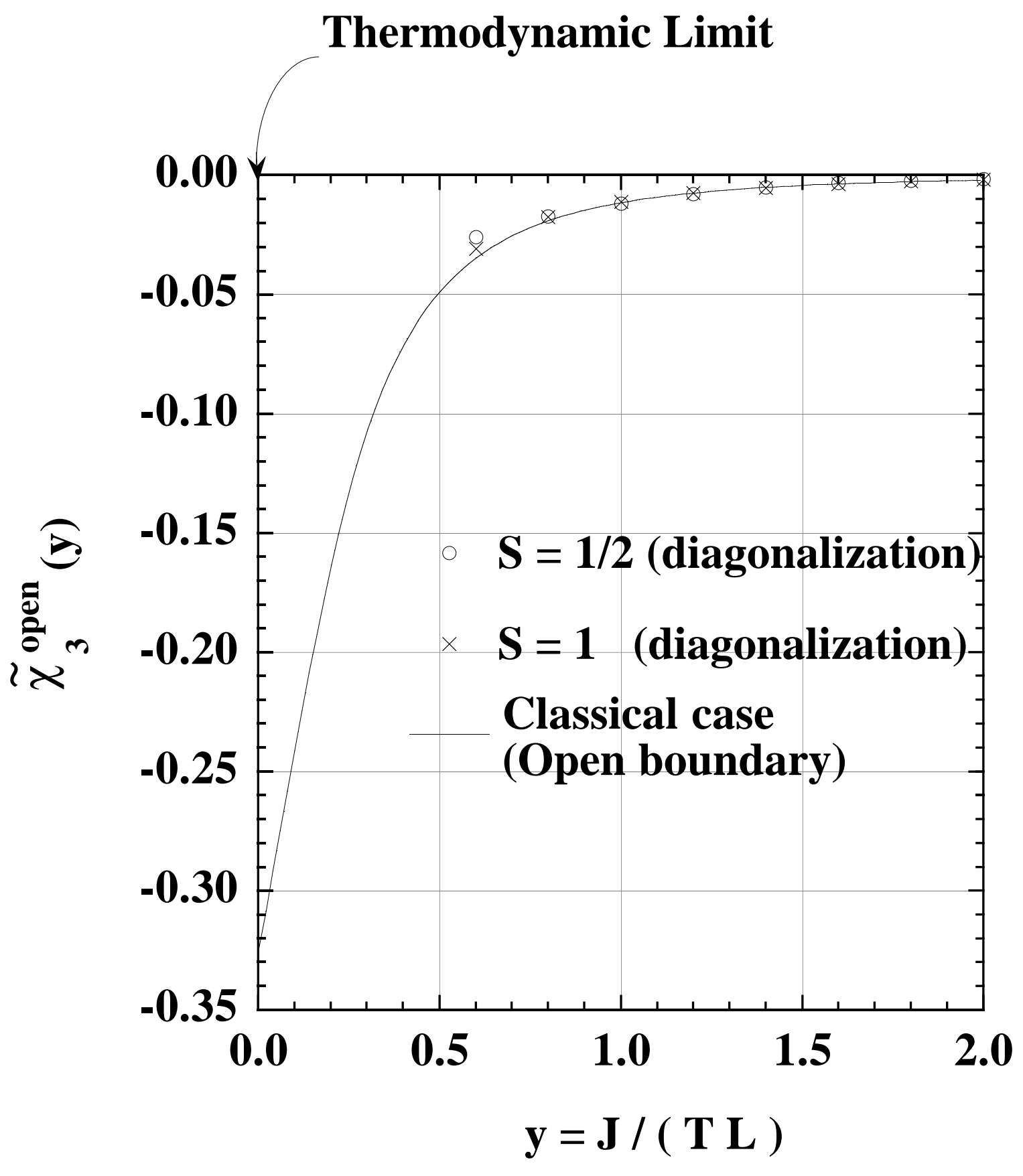

Fig.8. H. Nakamura, N. Hatano \& M. Takahashi 